\title{
Unified treatment of coupled optical and acoustic phonons in piezoelectric cubic materials
}

\author{
Willatzen, Morten; Wang, Zhong Lin
}

Published in:

Physical Review B

Link to article, DOI:

10.1103/PhysRevB.92.224101

Publication date:

2015

Document Version

Publisher's PDF, also known as Version of record

Link back to DTU Orbit

Citation (APA):

Willatzen, M., \& Wang, Z. L. (2015). Unified treatment of coupled optical and acoustic phonons in piezoelectric cubic materials. Physical Review B, 92, [224101]. https://doi.org/10.1103/PhysRevB.92.224101

\section{General rights}

Copyright and moral rights for the publications made accessible in the public portal are retained by the authors and/or other copyright owners and it is a condition of accessing publications that users recognise and abide by the legal requirements associated with these rights.

- Users may download and print one copy of any publication from the public portal for the purpose of private study or research.

- You may not further distribute the material or use it for any profit-making activity or commercial gain

- You may freely distribute the URL identifying the publication in the public portal

If you believe that this document breaches copyright please contact us providing details, and we will remove access to the work immediately and investigate your claim. 


\title{
Unified treatment of coupled optical and acoustic phonons in piezoelectric cubic materials
}

\author{
Morten Willatzen \\ Technical University of Denmark, Department of Photonics Engineering, Kgs-Lyngby 2800, Denmark \\ and Beijing Institute of Nanoenergy and Nanosystems, Chinese Academy of Sciences, Beijing, China \\ Zhong Lin Wang \\ School of Materials Science \& Engineering Georgia Institute of Technology, Atlanta, Georgia 30332, USA \\ and Beijing Institute of Nanoenergy and Nanosystems, Chinese Academy of Sciences, Beijing, China
}

(Received 21 September 2015; published 1 December 2015)

\begin{abstract}
A unified treatment of coupled optical and acoustic phonons in piezoelectric cubic materials is presented whereby the lattice displacement vector and the internal ionic displacement vector are found simultaneously. It is shown that phonon couplings exist in pairs only; either between the electric potential and the lattice displacement coordinate perpendicular to the phonon wave vector or between the two other lattice displacement components. The former leads to coupled acousto-optical phonons by virtue of the piezoelectric effect. We then establish three new conjectures that entirely stem from piezoelectricity in a cubic structured material slab. First, it is shown that isolated optical phonon modes generally cannot exist in piezoelectric cubic slabs. Second, we prove that confined acousto-optical phonon modes only exist for a discrete set of in-plane wave numbers in piezoelectric cubic slabs. Third, it is shown that coupled acousto-optical phonons do not exist at the longitudinal-optical (LO) phonon frequency where the dielectric constant vanishes.
\end{abstract}

DOI: 10.1103/PhysRevB.92.224101

PACS number(s): 63.20.-e, 77.65.-j

\section{INTRODUCTION}

Optical and acoustic phonons play a key role in the dissipation mechanisms of quantum electronic systems and determine the thermal and electrical properties of solids. While electron-phonon interactions usually lead to performance degradation of devices, they may also open up possibilities to tune device characteristics by virtue of material and geometry design and appropriate control of ambient properties as well as external parameters. Recent examples of phonon tuning applications include the realization of population inversion in InGaAs quantum dot systems [1-3] and phonon-assisted gain in semiconductor quantum dot masers [4-6]. It was demonstrated in Refs. [7,8] that phonon reservoirs significantly influence dephasing mechanisms [9-11] in quantum dot cavity systems with strong implications for potential applications in quantum information technology (QIT) [12]. Dephasing is a limiting factor for QIT since it destroys the entanglement between light and matter [7]. Some other recent phenomena where optical and acoustic phonon interactions are central include dissipation mechanisms in graphene and consequences for new graphene-based electronic and optical devices [13-15].

Piezoelectricity is important for applications and the understanding of phonon interactions in $3 \mathrm{D}$ and $2 \mathrm{D}$ semiconductor materials lacking inversion symmetry. A recent discussion of piezoelectric applications in 2D (monolayer) $\mathrm{MoS}_{2}$ can be found in Ref. [16]. Acoustic phonon limited mobility in 2D $\mathrm{MoS}_{2}$ was addressed theoretically in Ref. [17]. Graphenebased devices using GaAs as a substrate reveal significant influence at low temperatures on relaxation mechanisms due to piezoelectric surface acoustic phonon scattering originating from the substrate [18-21]. Another example is piezoelectric surface acoustic generation and manipulation that show promising potential in both quantum phononics [22,23] and plasmonics [24,25].

In this paper we present a detailed treatment of phonons in semiconductors and a full accounting of crystal anisotropy and inversion-asymmetry effects including piezoelectricity for structures based on, e.g., zincblende or wurtzite symmetries such as GaAs, GaN, and $\mathrm{ZnO}$ materials or 2D materials including $\mathrm{MoS}_{2}$ and $\mathrm{BN}$ [26]. It is demonstrated in a first unified approach that optical and acoustic phonons are inherently coupled in piezoelectric materials, and we compare with the existing literature in the field where optical and acoustic phonons are treated separately [27-31]. This coupling of optical and acoustic phonons is important for the understanding of electron-phonon interaction selection rules in piezoelectric materials and, e.g., Raman scattering processes. By solving the fully coupled system we obtain, in one step, the acoustic and optical phonon modes and determine to what extent they are coupled. It will be shown that in piezoelectric cubic materials acoustic phonon couplings exist entirely between two of the lattice displacement coordinates $\left(u_{x}\right.$ and $\left.u_{z}\right)$ similar to the case of nonpiezoelectric cubic materials. An additional pair coupling is shown to exist between the third lattice displacement coordinate $\left(u_{y}\right)$ and the electric potential $(\phi)$ by virtue of the piezoelectric effect. Hence optical phonons are generally always coupled to acoustic phonons. Choosing a cubic piezoelectric slab as an example, we demonstrate that only coupled acousto-optical phonon modes are allowed unless the phonon in-plane wave-number component vanishes. We show that coupled acousto-optical phonons cannot exist at the LO phonon frequency where the dielectric constant vanishes. Further, it is proven that confined acousto-optical phonon modes cannot exist at any frequency in a piezoelectric slab. These findings are both qualitatively and quantitatively different from the standard nonpiezoelectric result. 


\section{THEORY}

From the elastic equations and the Maxwell-Poisson equation of a piezoelectric cubic material, we obtain the following set of differential equations in the lattice displacements $u_{x}, u_{y}, u_{z}$ and the electric potential $\phi[32]$ :

$$
\begin{gathered}
\left(-q_{x}^{2} c_{11}+c_{44} \frac{\partial^{2}}{\partial z^{2}}+\rho \omega^{2}\right) u_{x}+i q_{x}\left(c_{12}+c_{44}\right) \frac{\partial u_{z}}{\partial z}=0 \\
\left(-q_{x}^{2} c_{44}+c_{44} \frac{\partial^{2}}{\partial z^{2}}+\rho \omega^{2}\right) u_{y}+2 i q_{x} e_{14} \frac{\partial \phi}{\partial z}=0 \\
i q_{x}\left(c_{12}+c_{44}\right) \frac{\partial u_{x}}{\partial z}+\left(-q_{x}^{2} c_{44}+c_{11} \frac{\partial^{2}}{\partial z^{2}}+\rho \omega^{2}\right) u_{z}=0 \\
2 i q_{x} e_{14} \frac{\partial u_{y}}{\partial z}+\epsilon_{11}\left(q_{x}^{2}-\frac{\partial^{2}}{\partial z^{2}}\right) \phi=0
\end{gathered}
$$

where $c_{11}, c_{12}, c_{44}$ are the three independent stiffness components and $e_{14}, \rho, \epsilon_{11}$, and $q_{x}$ are the piezoelectric constant, the mass density, the permittivity, and the phonon wave-number component in the slab plane, respectively. We have followed the common choice where the $x$ axis is directed along the phonon in-plane wave-number component, i.e., $q_{y}=0$ [29].

It is clear from the structure of the differential equations above that two solution types (I and II) for $u_{x}$ and $u_{z}$ are possible. For the type-I modes, we have [apart from a factor $\exp (i \omega t)]$

$$
u_{x}=\sum_{j=1}^{2} A_{x, j} \cos \gamma_{j} z, \quad u_{z}=\sum_{j=1}^{2} A_{z, j} \sin \gamma_{j} z
$$

and for type-II modes

$$
u_{x}=\sum_{j=1}^{2} B_{x, j} \sin \gamma_{j} z, \quad u_{z}=\sum_{j=1}^{2} B_{z, j} \cos \gamma_{j} z .
$$

The above two types correspond to compressional (dilatational) and flexural modes, respectively, and they are known as Rayleigh-Lamb modes in the case of an isotropic slab.

Similarly, two solution forms exist for acousto-optical phonon modes. For type-I modes

$$
\begin{gathered}
u_{y}=A_{y, 1} \cos \left(\delta_{1} z\right)+A_{y, 2} \cosh \left(\delta_{2} z\right), \\
\phi=A_{\phi, 1} \sin \left(\delta_{1} z\right)+A_{\phi, 2} \sinh \left(\delta_{2} z\right),
\end{gathered}
$$

and for type-II modes

$$
\begin{aligned}
& u_{y}=B_{y, 1} \sin \left(\delta_{1} z\right)+B_{y, 2} \sinh \left(\delta_{2} z\right), \\
& \phi=B_{\phi, 1} \cos \left(\delta_{1} z\right)+B_{\phi, 2} \cosh \left(\delta_{2} z\right)
\end{aligned}
$$

We note that the phonon wave vector $z$ components $\gamma_{j}$ and $\delta_{j}(j=1,2)$ are obtained from the secular equation of the differential equation system in Eqs. (1)-(4) [32]. Combining
Eqs. (1) and (5) we obtain for the type-I acoustic modes

$$
A_{z, j}=\frac{c_{44} \gamma_{j}^{2}+q_{x}^{2} c_{11}-\rho \omega^{2}}{i \gamma_{j} q_{x}\left(c_{12}+c_{44}\right)} A_{x, j} \quad(j=1,2) .
$$

For the slab problem with boundaries at $\pm a / 2$ the stress tensor fulfills

$$
T_{x z}(z= \pm a / 2)=0, \quad T_{z z}(z= \pm a / 2)=0 .
$$

Use of the constitutive equations and Eq. (5) gives for type-I modes

$$
\begin{aligned}
& \sum_{j=1}^{2}-\gamma_{j} A_{x, j} \sin \left(\gamma_{j} \frac{a}{2}\right)+i q_{x} A_{z, j} \sin \left(\gamma_{j} \frac{a}{2}\right)=0 \\
& \sum_{j=1}^{2} i q_{x} c_{12} A_{x, j} \cos \left(\gamma_{j} \frac{a}{2}\right)+\gamma_{j} c_{11} A_{z, j} \cos \left(\gamma_{j} \frac{a}{2}\right)=0 .
\end{aligned}
$$

Solving the $4 \times 4$ determinental equation in the four unknowns $A_{x, 1}, A_{x, 2}, A_{z, 1}, A_{z, 2}$ leads to the dispersion relations $\left(q_{x}, \omega_{n}\right)$ for type-I acoustic phonons as well as the coupled $\left(u_{x}, u_{z}\right)$ phonon modes. A similar procedure can be used to determine the type-II phonon modes.

Next, consider the coupled $u_{y}-\phi$ phonon modes. Combining instead Eqs. (4), (7), and (8), we obtain for the type-I modes acousto-optical phonon modes

$$
\begin{aligned}
& 2 i q_{x} e_{14}\left[-\delta_{1} A_{y, 1} \sin \left(\delta_{1} z\right)+\delta_{2} A_{y, 2} \sinh \left(\delta_{2} z\right)\right] \\
& \quad+\epsilon_{11}\left(q_{x}^{2}+\delta_{1}^{2}\right) A_{\phi, 1} \sin \left(\delta_{1} z\right) \\
& \quad+\epsilon_{11}\left(q_{x}^{2}-\delta_{2}^{2}\right) A_{\phi, 2} \sinh \left(\delta_{2} z\right)=0 \quad(j=1,2) .
\end{aligned}
$$

The appropriate acoustic boundary condition is

$$
T_{y z}(z= \pm a / 2)=c_{44} \frac{\partial u_{y}}{\partial z}+\left.e_{14} \frac{\partial \phi}{\partial x}\right|_{z= \pm a / 2}=0,
$$

giving

$$
\begin{aligned}
& -c_{44} \delta_{1} A_{y, 1} \sin \left(\delta_{1} \frac{a}{2}\right)+c_{44} \delta_{2} A_{y, 2} \sinh \left(\delta_{2} \frac{a}{2}\right) \\
& +e_{14} i q_{x} A_{\phi, 1} \sin \left(\delta_{1} \frac{a}{2}\right)+e_{14} i q_{x} A_{\phi, 2} \sinh \left(\delta_{2} \frac{a}{2}\right)=0 .
\end{aligned}
$$

Two electric boundary conditions are needed since the electric field outside the slab must be taken into account as well. For vacuum environments, the Maxwell-Poisson equation simply reads, if $|z|>a / 2$,

$$
-\epsilon_{0} \nabla^{2} \phi=\epsilon_{0}\left(q_{x}^{2}-\frac{\partial^{2}}{\partial z^{2}}\right) \phi=0
$$

with the solution

$$
\begin{aligned}
& \phi(z)=\phi_{+} e^{-\left|\mathbf{q}_{\|}\right| z} e^{i \mathbf{q}_{\|} \cdot \mathbf{r}} \quad \text { if } z>a / 2, \\
& \phi(z)=\phi_{-} e^{\left|\mathbf{q}_{\|}\right| z} e^{i \mathbf{q}_{\|} \cdot \mathbf{r}} \quad \text { if } z<-a / 2 .
\end{aligned}
$$

Continuity of the transverse electric field at $z= \pm a / 2$ yields for type-I acousto-optical phonon modes

$$
\phi_{+}=\frac{A_{\phi, 1} \sin \left(\delta_{1} \frac{a}{2}\right)+A_{\phi, 2} \sinh \left(\delta_{2} \frac{a}{2}\right)}{e^{-\left|\mathbf{q}_{\|}\right| a / 2}}, \quad \phi_{-}=-\phi_{+} .
$$


Imposing continuity of the normal electric displacement at the slab boundaries gives for type-I modes

$$
\begin{aligned}
& -\epsilon_{11}\left[\delta_{1} A_{\phi, 1} \cos \left(\delta_{1} \frac{a}{2}\right)+\delta_{2} A_{\phi, 2} \cosh \left(\delta_{2} \frac{a}{2}\right)\right] \\
& +e_{14} i q_{x}\left[A_{y, 1} \cos \left(\delta_{1} \frac{a}{2}\right)+A_{y, 2} \cosh \left(\delta_{2} \frac{a}{2}\right)\right] \\
& =\epsilon_{0} \phi_{+}\left|\mathbf{q}_{\|}\right| e^{-\left|\mathbf{q}_{\|}\right| a / 2} .
\end{aligned}
$$

The above set of equations completely specifies the type-I acousto-optical phonon modes. We note that the procedure for determining type-II modes goes along the same steps. Since the electric field $\mathbf{E}=-\nabla \phi$, the ionic displacement $\mathbf{u}_{\text {ion }}=\mathbf{u}_{+}-\mathbf{u}_{-}$ can be found from the Born-Huang equations [33]

$$
\mathbf{u}_{\mathrm{ion}}=-\sqrt{\frac{\Omega}{m_{r}}} \frac{\sqrt{\epsilon(0)-\epsilon(\infty)} \omega_{T O}}{\omega^{2}-\omega_{T O}^{2}} \mathbf{E},
$$

where $\Omega$ and $m_{r}$ are the unit cell volume and reduced ion mass, respectively. Hence, the present coupled phonon formalism allows determination of the lattice displacement $\mathbf{u}$ and the internal ion displacement $\mathbf{u}_{\text {ion }}$ simultaneously.

Finally, to completely specify the phonon mode solutions, coupled normalization conditions are needed:

$$
\begin{aligned}
& \int d \mathbf{r}\left[\sqrt{\frac{m_{r}}{\Omega}} \mathbf{u}_{\text {ion }}^{*}(\mathbf{r}, t)\right]\left[\sqrt{\frac{m_{r}}{\Omega}} \mathbf{u}_{\text {ion }}(\mathbf{r}, t)\right] \\
& +\int d \mathbf{r}\left[\sqrt{\rho} \mathbf{u}^{*}(\mathbf{r}, t)\right][\sqrt{\rho} \mathbf{u}(\mathbf{r}, t)]=\frac{\hbar}{2 \omega_{n}},
\end{aligned}
$$

where $\omega_{n}$ is the phonon mode frequency. An important result that distinguishes piezoelectric materials from nonpiezoelectric materials can now be proved. We will show that at the LO phonon frequency where the permittivity is zero coupled acousto-optical phonon modes cannot be excited. In contrast, in nonpiezoelectric media confined optical phonon modes with an arbitrary in-plane wave number $q_{x}$ exist at the LO phonon frequency.

First, if $\epsilon_{11}=0$, Eq. (4) shows that

$$
u_{y}=A_{y} e^{i q_{x} x} .
$$

Then Eqs. (2) and (16) yield

$$
\phi=\left(\phi_{0}+\phi_{1} z\right) e^{i q_{x} x}, \quad \phi_{0}=\phi_{1}=0 .
$$

Continuity of the transverse electric field component guarantees further that

$$
\phi_{+}=\phi_{-}=0 \text {, }
$$

and finally, from continuity in the normal electric displacement, we obtain

$$
A_{y}=0 .
$$

Hence, both the electric potential $\phi$ and the phonon $u_{y}$ component must be zero simultaneously. In other words, it is not possible to excite coupled $u_{y}-\phi$ modes at the LO phonon frequency. It is well known that confined LO phonon modes exist at the LO phonon frequency. We will now prove that confined coupled acousto-optical $u_{y}-\phi$ modes cannot exist in piezoelectric media except at certain discrete $q_{x}$ wave-number values.
A confined phonon mode is characterized by an electric field that vanishes at the slab interfaces. Thus, coupled $u_{y}-\phi$ solutions can be sought quite generally in the form (type I modes)

$$
u_{y}=A_{y} \cos \left(\frac{m \pi}{a} z\right) e^{i q_{x} x}, \quad \phi=A_{\phi} \sin \left(\frac{m \pi}{a} z\right) e^{i q_{x} x},
$$

where $m=2,4,6, \ldots$. From Eq. (2) we have

$$
\left(-q_{x}^{2} c_{44}-\left(\frac{m \pi}{a}\right)^{2} c_{44}+\rho \omega^{2}\right) A_{y}+2 i q_{x} e_{14}\left(\frac{m \pi}{a}\right) A_{\phi}=0,
$$

and from Eq. (4)

$$
-2 i q_{x} e_{14}\left(\frac{m \pi}{a}\right) A_{y}+\epsilon_{11}\left(q_{x}^{2}+\left(\frac{m \pi}{a}\right)^{2}\right) A_{\phi}=0 .
$$

Hence the possible mode frequencies are

$\omega_{m}=\sqrt{\frac{c_{44}}{\rho}}\left[q_{x}^{2}+\left(\frac{m \pi}{a}\right)^{2}+\frac{4 q_{x}^{2} e_{14}^{2}\left(\frac{m \pi}{a}\right)^{2}}{\epsilon_{11} c_{44}\left(q_{x}^{2}+\left(\frac{m \pi}{a}\right)^{2}\right)}\right]^{1 / 2}$.

It follows immediately from the preceding equations that Eq. (16) is fulfilled. Continuity in the transverse electric field and normal displacement yields $\phi_{+}=0$ and

$$
A_{\phi}=\frac{i q_{x} e_{14}}{\epsilon_{11}(-1)^{m / 2}\left(\frac{m \pi}{a}\right)} A_{y} .
$$

Combining Eqs. (31) and (33) requires

$$
2(-1)^{m / 2}\left(\frac{m \pi}{a}\right)^{2}=q_{x}^{2}+\left(\frac{m \pi}{a}\right)^{2},
$$

which is possible only at certain discrete $q_{x}$ values $\left\{q_{x, m}\right\}$. Hence we have proven the conjecture that confined coupled $u_{y}-\phi$ phonon modes cannot exist in piezoelectric media except at certain discrete wave-number values, in contrast to the case of nonpiezoelectric media where confined optical phonon modes exist for any in-plane wave number $q_{x}$.

\section{DISCUSSIONS}

Consider first the influence of anisotropy on the phonon dispersion for the coupled $u_{x}-u_{z}$ lattice displacement components in a cubic piezoelectric GaAs slab. The physical properties of GaAs are $c_{11}=11.88 \times 10^{10} \mathrm{~Pa}, c_{12}=$ $5.38 \times 10^{10} \mathrm{~Pa}, c_{44}=5.94 \times 10^{10} \mathrm{~Pa}, \rho=5.318 \times 10^{3} \mathrm{~kg} / \mathrm{m}^{3}$, $e_{14}=0.154 \mathrm{C} / \mathrm{m}^{2}, \quad \epsilon(0)=12.9 \epsilon_{0}, \hbar \omega_{L O}=33.2 \mathrm{meV}$, $\hbar \omega_{T O}=36.1 \mathrm{meV}, \epsilon_{\infty}=\epsilon(0) \frac{\omega_{T O}^{2}}{\omega_{L O}^{2}}$.

Since all cubic materials are anisotropic, the isotropic relation

$$
c_{44}=\frac{c_{11}-c_{12}}{2}
$$

used for GaAs in, e.g., Ref. [29] is an approximation. In fact, the degree of anisotropy, defined as

$$
\alpha=\left|\frac{\frac{c_{11}-c_{12}}{2}-c_{44}}{c_{44}}\right|,
$$



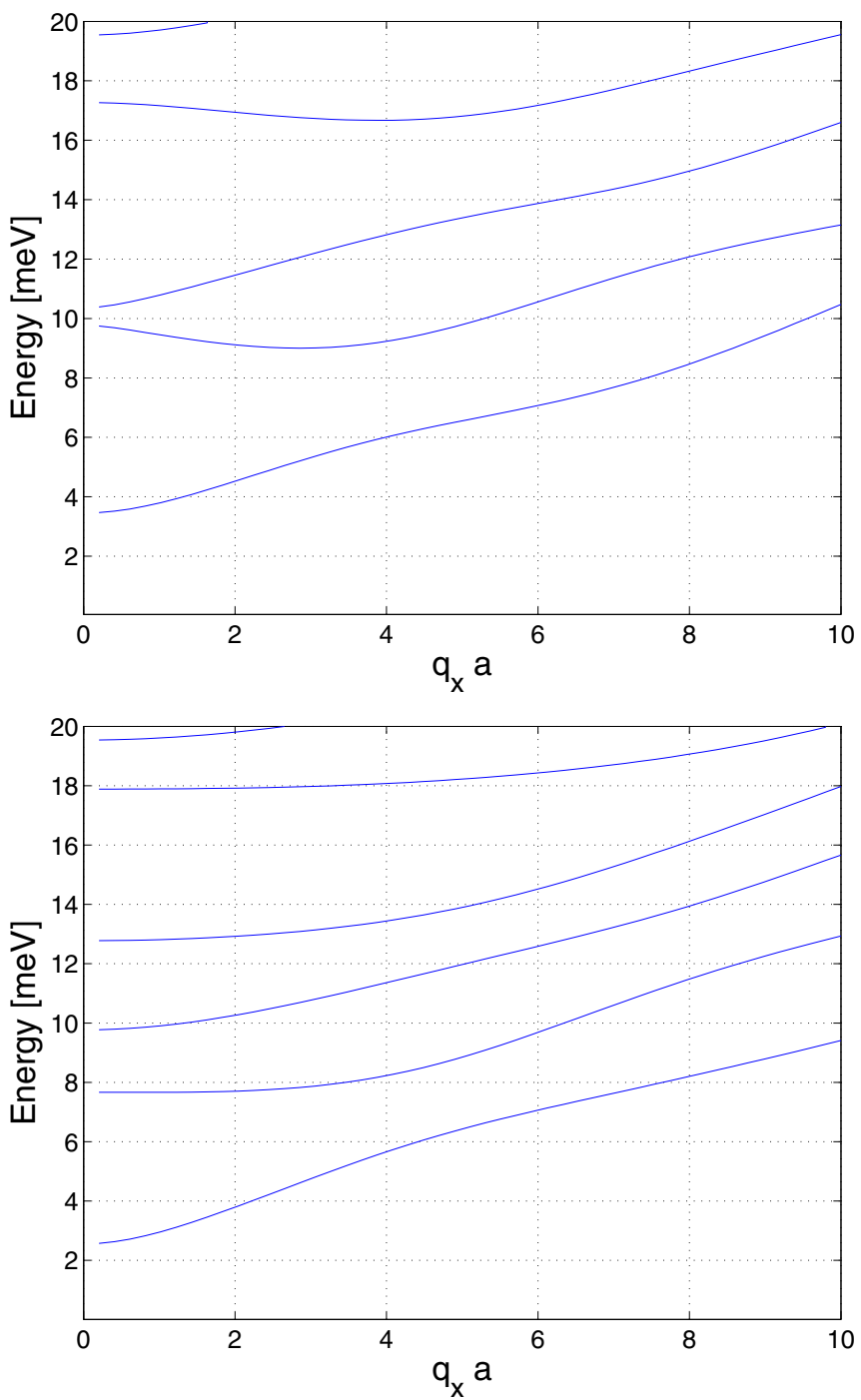

FIG. 1. (Color online) Dispersion relations for the coupled $u_{x}-u_{z}$ acoustic phonon modes of a $2 \mathrm{~nm} \mathrm{GaAs} \mathrm{slab.} \mathrm{(Upper} \mathrm{plot)}$ $\mathrm{GaAs}$ and (lower plot) GaAs but using the isotropic assumption: $c_{44}=\frac{c_{11}-c_{12}}{2}=3.25 \times 10^{10} \mathrm{~Pa}$. The first axis is $q_{x} a$ and the second axis the phonon band energy in $\mathrm{meV}$.

is large in GaAs and equal to $45 \%$. Hence, substantial deviations in the phonon modes and dispersion relations due to anisotropy must be expected for GaAs. In passing, we note that it follows from the results above that unit-cell inversion asymmetry and piezoelectricity do not influence the $u_{x}-u_{z}$ phonon modes and dispersion.

In Fig. 1 the coupled $u_{x}-u_{z}$ dispersion curves are shown for a $2 \mathrm{~nm}$ GaAs slab. Evidently, the phonon mode energies change markedly when including the full anisotropy (by more than $40 \%$ for the first band energy at $q_{x} a=0$ ) and so does the shape of the dispersion curves.

Next, let us consider the dispersion curves for coupled $u_{y}-\phi$ acousto-optical phonon modes.

In Fig. 2 the $u_{y}-\phi$ dispersion curves are shown for a $2 \mathrm{~nm}$ GaAs slab. It is evident that the coupled acousto-optical phonon modes are strongly influenced by anisotropy, as in the case with the coupled acoustic $u_{x}-u_{z}$ phonons, leading
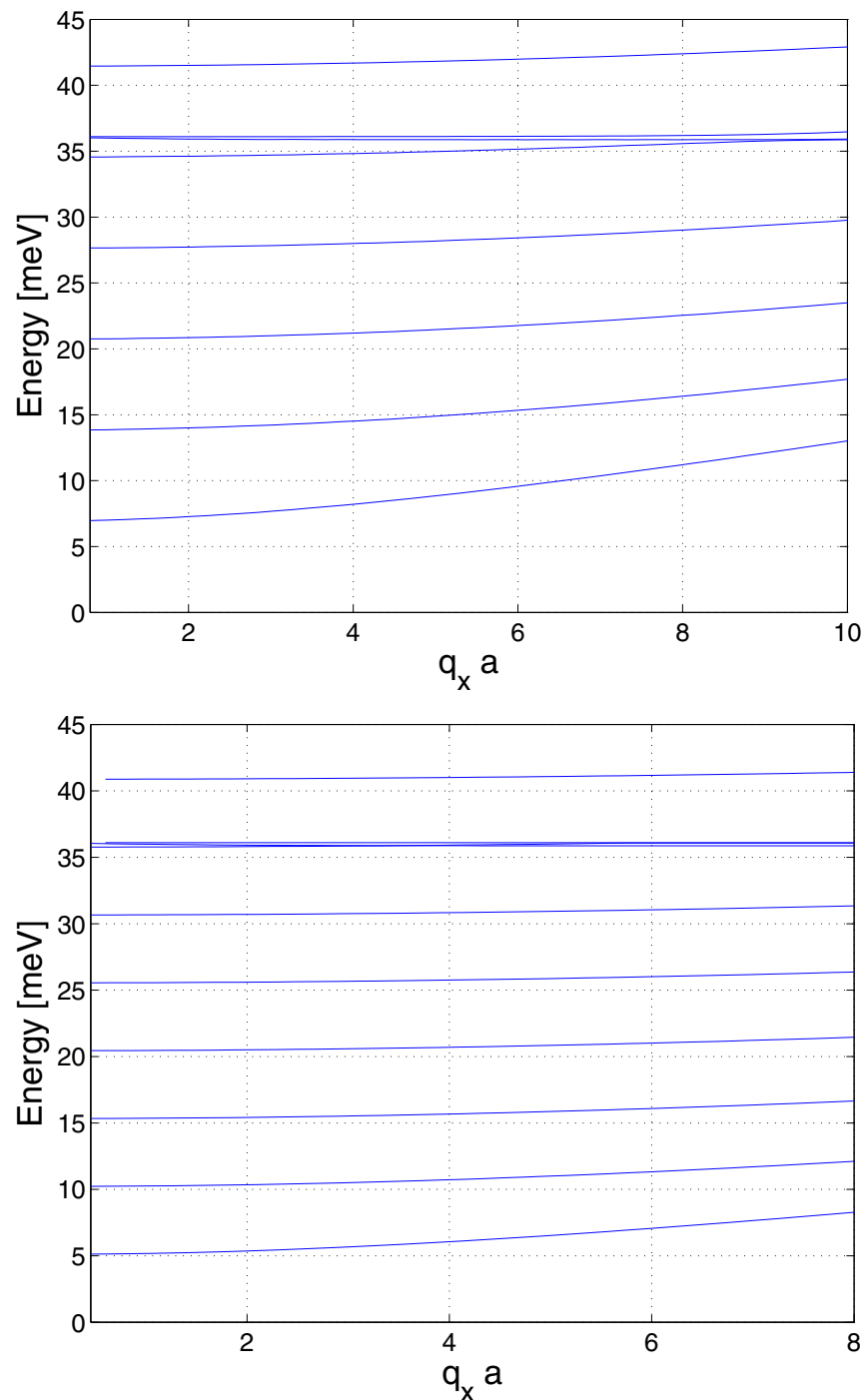

FIG. 2. (Color online) Dispersion relations for the coupled $u_{y}-\phi$ acousto-optical phonon modes of a $2 \mathrm{~nm}$ GaAs slab. (Upper plot) GaAs including piezoelectricity and anisotropy and (lower plot) GaAs but using the isotropic non-piezoelectric assumption: $c_{44}=\frac{c_{11}-c_{12}}{2}=3.25 \times 10^{10} \mathrm{~Pa}$ and $e_{14}=0$.

to a difference of about $40 \%$ in the first band energy near $q_{x} a=0$. Moreover, a close inspection of the dispersion curves around the LO phonon energy of $36.1 \mathrm{meV}$ reveals that, when piezoelectricity is included and for any strictly positive $q_{x} a$ value, no band crosses the forbidden LO phonon energy, in agreement with the proof given above.

It must be noted that interface phonons are not captured in the plots as they correspond to imaginary $q_{x}$ values, but the procedure for finding them is the same as for nonpiezoelectric and isotropic media [27].

A combined treatment of coupled acoustic and optical phonons in piezoelectric cubic media was presented that allows determination of the lattice displacement vector and the internal ionic displacement vector simultaneously. It was demonstrated that phonons generally exist in pairs due to couplings between either the lattice displacement component perpendicular to the phonon wave vector $\left(u_{y}\right.$ coordinate) and 
the electric potential $\phi$ or the two other lattice displacement components $\left(u_{x}\right.$ and $\left.u_{z}\right)$. Three new conjectures were derived for piezoelectric cubic slabs: (1) isolated optical phonon modes cannot exist unless the in-plane phonon wave number vanishes $\left(q_{x}=0\right)$; (2) we proved that confined acousto-optical phonon modes only exist in piezoelectric cubic slabs for a discrete set of in-plane wave numbers; (3) at the LO phonon frequency coupled acousto-optical phonons cannot exist. We point out that the present method can be generalized along the same lines to other piezoelectric materials such as piezoelectric hexagonal (wurtzite) materials.

\section{ACKNOWLEDGMENT}

The authors are grateful to Professor Antti-Pekka Jauho for comments and a critical reading of the manuscript.
[1] J. H. Quilter, A. J. Brash, F. Liu, M. Glässl, A. M. Barth, V. M. Axt, A. J. Ramsay, M. S. Skolnick, and A. M. Fox, Phys. Rev. Lett. 114, 137401 (2015).

[2] A. E. Siegman, Lasers (University Science Books, Oxford, 1986).

[3] M. Glässl, A. M. Barth, and V. M. Axt, Phys. Rev. Lett. 110, 147401 (2013).

[4] M. J. Gullans, Y.-Y. Liu, J. Stehlik, J. R. Petta, and J. M. Taylor, Phys. Rev. Lett. 114, 196802 (2015).

[5] M. Sargent, M. Scully, and W. Lamb, Laser Physics (Perseus Books Group, New York, 1978).

[6] Y. Y. Liu, J. Stehlik, C. Eichler, M. J. Gullans, J. M. Taylor, and J. R. Petta, Science 347, 285 (2015).

[7] P. Kaer, T. R. Nielsen, P. Lodahl, A.-P. Jauho, and J. Mørk, Phys. Rev. Lett. 104, 157401 (2010).

[8] A. Nysteen, P. Kaer, and J. Mørk, Phys. Rev. Lett. 110, 087401 (2013).

[9] A. Aufféves, J. M. Gerard, and J. P. Poizat, Phys. Rev. A 79, 053838 (2009).

[10] T. Yoshie, A. Scherer, J. Heindrickson, G. Khitrova, H. M. Gibbs, G. Rupper, C. Ell, O. B. Shchekin, and D. G. Deppe, Nature (London) 432, 200 (2004).

[11] H. J. Carmichael, R. J. Brecha, M. G. Raizen, H. J. Kimble, and P. R. Rice, Phys. Rev. A 40, 5516 (1989).

[12] E. Knill, R. Laflamme, and G. J. Milburn, Nature (London) 409, 46 (2001).

[13] M. W. Graham, S.-F. Shi, D. C. Ralph, J. Park, and P. L. McEuen, Nat. Phys. 9, 103 (2009).

[14] F. D. Natterer, Y. Zhao, J. Wyrick, Y.-H. Chan, W.-Y. Ruan, M.-Y. Chou, K. Watanabe, T. Taniguchi, N. B. Zhitenev, and J. A. Stroscio, Phys. Rev. Lett. 114, 245502 (2015).

[15] D. N. Basov, M.M. Fogler, A. Lanzara, F. Wang, and Y. Zhang, Rev. Mod. Phys. 86, 959 (2014).
[16] W. Wu, L. Wang, Y. Li, F. Zhang, L. Lin, S. Niu, D. Chenet, X. Zhang, Y. Hao, T. F. Heinz, J. Hone, and Z. L. Wang, Nature (London) 514, 470 (2014).

[17] K. Kaasbjerg, K. S. Thygesen, and A.-P. Jauho, Phys. Rev. B 87, 235312 (2013).

[18] S. H. Zhang, W. Xu, F. M. Peeters, and S. M. Badalyan, Phys. Rev. B 89, 195409 (2014).

[19] M. Woszczyna, M. Friedemann, M. Gtz, E. Pesel, K. Pierz, T. Weimann, and F. J. Ahlers, Appl. Phys. Lett. 100, 164106 (2012).

[20] S. Fratini and F. Guinea, Phys. Rev. B 77, 195415 (2008).

[21] V. Perebeinos, S. V. Rotkin, A. G. Petrov, and P. Avouris, Nano Lett. 9, 312 (2009).

[22] A. Clerk, Nat. Phys. 8, 256 (2012).

[23] M. V. Gustafsson, P. V. Santos, G. Johansson, and P. Delsing, Nat. Phys. 8, 338 (2012).

[24] M. Farhat, S. Guenneau, and H. Bagci, Phys. Rev. Lett. 111, 237404 (2013)

[25] J. Schiefele, J. Pedros, F. Sols, F. Calle, and F. Guinea, Phys. Rev. Lett. 111, 237405 (2013).

[26] K. H. Michel and B. Verberck, Phys. Rev. B 80, 224301 (2009).

[27] J. J. Licari and R. Evrard, Phys. Rev. B 15, 2254 (1977).

[28] K. L. Kliewer and R. Fuchs, Phys. Rev 144, 495 (1966); 150, 573 (1966).

[29] N. Bannov, V. Aristov, V. Mitin, and M. A. Stroscio, Phys. Rev. B 51, 9930 (1995).

[30] B. K. Ridley, Electrons and Phonons in Semiconductor Multilayers, 2nd ed. (Cambridge University Press, New York, 2009).

[31] M. A. Stroscio and M. Dutta, Phonons in Nanostructures (Cambridge University Press, Cambridge, 2001).

[32] Details will be presented elsewhere.

[33] M. Born and K. Huang, Dynamical Theory of Crystal Lattices (Oxford University Press, Oxford, 1954). 